PROCEEDINGS OF THE

AMERICAN MATHEMATICAL SOCIETY

Volume 127, Number 6, Pages 1837-1845

S 0002-9939(99)04665-1

Article electronically published on February 23, 1999

\title{
A CENTRAL LIMIT THEOREM FOR MARKOV CHAINS AND APPLICATIONS TO HYPERGROUPS
}

\author{
LÉONARD GALLARDO
}

(Communicated by Stanley Sawyer)

\begin{abstract}
Let $\left(X_{n}\right)$ be a homogeneous Markov chain on an unbounded Borel subset of $\mathbb{R}$ with a drift function $d$ which tends to a limit $m_{1}$ at infinity. Under a very simple hypothesis on the chain we prove that $n^{-1 / 2}\left(X_{n}-\sum_{k=1}^{n} d\left(X_{k-1}\right)\right)$ converges in distribution to a normal law $N\left(0, \sigma^{2}\right)$ where the variance $\sigma^{2}$ depends on the asymptotic behaviour of $\left(X_{n}\right)$. When $d-m_{1}$ goes to zero quickly enough and $m_{1} \neq 0$, the random centering may be replaced by $n m_{1}$. These results are applied to the case of random walks on some hypergroups.
\end{abstract}

\section{INTRODUCTION}

In the sequel, $\left(X_{n}\right)$ is a homogeneous Markov chain on an unbounded Borel subset $K$ of $\mathbb{R}$. Let $\alpha>0$. We consider the following functions:

$$
\begin{aligned}
d(t) & =\mathbb{E}\left(X_{n}-X_{n-1} \mid X_{n-1}=t\right), \\
c_{\alpha}(t) & =\mathbb{E}\left(\left|X_{n}-X_{n-1}\right|^{\alpha} \mid X_{n-1}=t\right),
\end{aligned}
$$

for $t \in K$. The first function $d$ is called the drift of $\left(X_{n}\right)$. When $\left(X_{n}\right)$ is a classical (non-deterministic) random walk on $\mathbb{R}$ with finite second moment, $d(t) \equiv$ $m_{1}$ and $c_{2}(t) \equiv m_{2}$ are constant functions. Then the Central Limit Theorem (CLT) asserts that $n^{-1 / 2}\left(X_{n}-n m_{1}\right)$ converges in distribution to the centered normal law $N\left(0, \sigma^{2}\right)$ with variance $\sigma^{2}=m_{2}-m_{1}^{2}$. In this paper we will generalize this result to certain Markov chains such that $c_{2}$ is a bounded function on $K$ and the limits $\lim _{|t| \rightarrow \infty} d(t)=m_{1}$ and $\lim _{|t| \rightarrow \infty} c_{2}(t)=m_{2}$ exist.

As in [G1] and [G3], where we established a law of large numbers, our results can be applied very naturally to get a CLT for random walks on one-dimensional hypergroups with asymptotic drift of the convolution (see section 4). Central limit theorems for random walks have already been studied in some particular commutative hypergroups using Fourier methods ([B.H]). The results of this paper are of a different nature. We emphasize the asymptotic behaviour of the Markov chain and derive a CLT using only tools from martingale theory and ergodic theory.

Received by the editors April 14, 1997 and, in revised form, September 22, 1997.

1991 Mathematics Subject Classification. Primary 60J10, 60F05, 60J15.

(C)1999 American Mathematical Society 


\section{Generalities AND hypotheses}

2.1. For the Markov chain $\left(X_{n}\right)$ we will make use of some of the following hypotheses:

$H_{0}(\alpha)=$ "function $c_{\alpha}$ exists and is measurable and bounded on $K$ ".

$H_{1}=$ " $H_{0}(1)$ is satisfied and $\lim _{|t| \rightarrow \infty} d(t)=m_{1}(\in \mathbb{R})$ exists".

$H_{2}=$ " $H_{0}(2)$ is satisfied and $\lim _{|t| \rightarrow \infty} c_{2}(t)=m_{2}\left(\in \mathbb{R}_{+}\right)$exists".

$H_{1}$ and $H_{2}$ are often verified in applications. In the case of random walks on hypergroups, a different condition is satisfied:

$H_{3}=$ "There exists a sequence $\left(\xi_{n}\right)$ of I.I.D random variables with finite second moment such that

$$
\left|X_{n}-X_{n-1}\right| \leq \xi_{n} \quad \text { a.s. }
$$

for all $n \in \mathbb{N}^{*}$."

Note that if $H_{0}(2)$ is satisfied, we have $c_{2}(t)-d^{2}(t) \geq 0 \quad(\forall t \in K)$. We will suppose that this function never vanishes, i.e., $\left(X_{n}\right)$ has no absorbing state.

\subsection{Definitions and notations.}

2.2.1. As usual, for every $x \in K$, we will denote by $\mathbb{P}_{x}$ (resp. $\mathbb{E}_{x}$ ) the probability (resp. the expectation) given $X_{0}=x$. In the same way, if $\nu$ is any probability measure on $K, \mathbb{P}_{\nu}$ (resp. $\mathbb{E}_{\nu}$ ) is the probability (resp. the expectation) given that the initial state $X_{0}$ is distributed according to $\nu$.

2.2.2. In the sequel when we suppose that $\left(X_{n}\right)$ is a recurrent chain, this means that:

i) If $K$ is a countable set, $\left(X_{n}\right)$ is an irreducible and recurrent chain on $K$ in the classical sense. In this case we will denote by $\lambda$ its invariant measure.

ii) If $K$ is an uncountable Borel subset of $\mathbb{R},\left(X_{n}\right)$ is Harris-recurrent with respect to an invariant Radon measure $\lambda$ supported by $K([\mathrm{R}])$.

In both cases, $\left(X_{n}\right)$ is said to be null recurrent if $\lambda(K)=+\infty$. Otherwise it is called positive recurrent and we can always assume that $\lambda$ is a probability measure.

2.2.3. The chain will be called transient if for some $x \in K,\left|X_{n}\right| \rightarrow+\infty \quad \mathbb{P}_{x} \quad$ a.s.

\section{The Central Limit Theorem}

We will break the result into two statements according to the asymptotic behaviour of the chain.

3.1. Theorem A. If $\left(X_{n}\right)$ is positive recurrent with invariant measure $\lambda$ and satisfies hypothesis $H_{0}(2)$, then

$$
n^{-1 / 2}\left(X_{n}-\sum_{k=1}^{n} d\left(X_{k-1}\right)\right) \stackrel{\mathcal{L}}{\longrightarrow} N\left(0, \sigma^{2}\right) \quad(n \rightarrow+\infty),
$$

where $\sigma^{2}=\int_{K}\left(c_{2}(t)-d^{2}(t)\right) \lambda(d t)$. 
3.2. Theorem B. If $\left(X_{n}\right)$ is null recurrent or transient, satisfies $H_{1}$ and $H_{2}$ with $\sigma^{2}=m_{2}-m_{1}^{2}>0$ and moreover satisfies either hypothesis $H_{0}(2+\epsilon)$ (for some $\epsilon>0)$ or hypothesis $\mathrm{H}_{3}$, then

$$
n^{-1 / 2}\left(X_{n}-\sum_{k=1}^{n} d\left(X_{k-1}\right)\right) \stackrel{\mathcal{L}}{\longrightarrow} N\left(0, \sigma^{2}\right) \quad(n \rightarrow+\infty) .
$$

(In both results, $\stackrel{\mathcal{L}}{\longrightarrow}$ means convergence in distribution.)

Proof. The beginning of the proof is common to (3.1) and (3.2). It is not difficult to verify that the sequence

$$
M_{n}=X_{n}-\sum_{k=1}^{n} d\left(X_{k-1}\right), \quad n \geq 1,
$$

is a martingale with respect to the filtration $\mathcal{F}_{n}=\sigma\left(X_{k} ; k \leq n\right)$. Let $Z_{k}=$ $M_{k}-M_{k-1}$ be the increment of this martingale. We easily get

$$
\mathbb{E}\left(Z_{k}^{2} \mid \mathcal{F}_{k-1}\right)=g\left(X_{k-1}\right) \quad \text { a.s. }
$$

where $g(t)=c_{2}(t)-d^{2}(t)$. For every $n \in \mathbb{N}, j \in \mathbb{N}, a>0$ and $\epsilon>0$, let us consider the following expressions:

$$
\begin{aligned}
V_{n}^{2} & =\sum_{k=1}^{n} g\left(X_{k-1}\right), \\
s_{n}^{2} & =\mathbb{E}\left(V_{n}^{2}\right)=\sum_{k=1}^{n} \mathbb{E}\left(Z_{k}^{2}\right), \\
W_{j}(a) & =\mathbb{E}\left(Z_{j}^{2} \mathbb{I}_{\left[\left|Z_{j}\right|>a\right]} \mid \mathcal{F}_{j-1}\right), \\
H_{n}^{\epsilon} & =\frac{1}{n} \sum_{j=1}^{n} W_{j}(\epsilon \sigma \sqrt{j}) .
\end{aligned}
$$

The theorems will be proved if we show that the CLT for martingales can be applied to $\left(M_{n}\right)$. For this purpose it is sufficient to prove the three following assertions $([\mathrm{H} . \mathrm{H}]$ or $[\mathrm{Br}])$ :

$$
\begin{gathered}
\lim _{n \rightarrow \infty} n^{-1} V_{n}^{2}=\sigma^{2} \quad \text { (in probability), } \\
\lim _{n \rightarrow \infty} n^{-1} s_{n}^{2}=\sigma^{2}, \\
\forall \epsilon>0, \lim _{n \rightarrow \infty} H_{n}^{\epsilon}=0 \quad \text { (in probability). }
\end{gathered}
$$

The last assertion is the Lindeberg condition. We now have to consider separately (3.1) and (3.2).

i) Proof of Theorem A. The bounded function $g$ is $\lambda$ integrable and the ergodic theorem gives immediately (3.9):

$$
n^{-1} V_{n}^{2}=\frac{1}{n} \sum_{k=1}^{n} g\left(X_{k-1}\right) \longrightarrow\langle\lambda, g\rangle=\sigma^{2} \quad \text { a.s. }
$$

But we have dominated convergence and (3.10) is also satisfied. For all fixed $a>0$, consider the function defined on $K$ by $h(x)=\mathbb{E}_{x}\left(Z_{1}^{2} \mathbb{I}_{\left[\left|Z_{1}\right|>a\right]}\right)$. By the 
Markov property and using notation (3.7), we have

$$
K_{n}(a):=\frac{1}{n} \sum_{j=1}^{n} h\left(X_{j-1}\right)=\frac{1}{n} \sum_{j=1}^{n} W_{j}(a) .
$$

But $h$ is also a bounded function on $K$ (indeed $h(x) \leq \mathbb{E}_{x}\left(Z_{1}^{2}\right) \leq 2 c_{2}(x)+2 d^{2}(x) \leq$ $\left.2\left(\left\|c_{2}\right\|_{\infty}+\left\|d^{2}\right\|_{\infty}\right)\right)$ and the ergodic theorem applied to $h$ gives

$$
\lim _{n \rightarrow \infty} K_{n}(a)=\langle\lambda, h\rangle=\mathbb{E}_{\lambda}\left(Z_{1}^{2} \mathbb{I}_{\left[\left|Z_{1}\right|>a\right]}\right)
$$

For every $a>0, \epsilon>0$, it is clear that we have

$$
\limsup _{n \rightarrow \infty} H_{n}^{\epsilon} \leq \lim _{n \rightarrow \infty} K_{n}(a) .
$$

This implies that the left-hand side of (3.13) is zero because $\lim _{a \rightarrow \infty} \mathbb{E}_{\lambda}\left(Z_{1}^{2} \mathbb{I}_{\left[\left|Z_{1}\right|>a\right]}\right)=$ 0 and assertion (3.11) follows.

ii) Proof of Theorem B. If we set $f(t)=g(t)-\sigma^{2}$, assertion (3.9) will follow immediately from the lemma:

3.14. Lemma. If $\left(X_{n}\right)$ is a null recurrent or transient chain on $K$ and if $f$ is a measurable and bounded function on $K$ such that $\lim _{|t| \rightarrow \infty} f(t)=0$, then

$$
\lim _{n \rightarrow \infty} \frac{1}{n} \sum_{i=1}^{n} f\left(X_{i}\right)=0 \text { a.s. }
$$

Proof of the lemma. If $\left(X_{n}\right)$ is transient, the result is trivial. If $\left(X_{n}\right)$ is null recurrent, let $\lambda$ be its invariant measure and let $E_{m}$ be an increasing sequence of subsets of $K$ such that $\bigcup_{m} E_{m}=K$ and $\lambda\left(E_{m}\right)<+\infty$ for all $m \in \mathbb{N}$. For all $k \in \mathbb{N}$, let us consider the function $f_{k}=\mathbb{I}_{[-k, k]} f$. By the Chacon-Ornstein theorem ([R], p. 123), we have

$$
\lim _{n \rightarrow \infty} \frac{\sum_{i=1}^{n}\left|f_{k}\left(X_{i}\right)\right|}{\sum_{i=1}^{n} \mathbb{I}_{E_{m}}\left(X_{i}\right)}=\frac{\left\langle\lambda,\left|f_{k}\right|\right\rangle}{\lambda\left(E_{m}\right)} \quad \text { a.s. },
$$

for all fixed $k$ and $m \in \mathbb{N}^{*}$. But $\lim _{m \rightarrow \infty} \lambda\left(E_{m}\right)=+\infty$, and we immediately deduce from (3.15) that

$$
\lim _{n \rightarrow \infty} \frac{1}{n} \sum_{i=1}^{n} f_{k}\left(X_{i}\right)=0 \quad \text { a.s. }
$$

for every $k \in \mathbb{N}^{*}$. The result of the lemma follows from the uniform convergence of $f_{k}$ to $f$ on $K$ as $k \rightarrow+\infty$.

The dominated convergence in (3.9) also implies (3.10). Now in order to prove (3.11) let us denote $b_{j}=\epsilon \sigma \sqrt{j}$ to simplify notations. We have to consider separately the two cases:

i) Suppose $H_{3}$ is satisfied. For every $j \geq 1$, we have

$$
Z_{j}^{2}=\left[\left(X_{j}-X_{j-1}\right)-d\left(X_{j-1}\right)\right]^{2} \leq 2\left(\xi_{j}^{2}+d^{2}\left(X_{j-1}\right)\right) .
$$

Then

$$
W_{j}\left(b_{j}\right) \leq 2\left(\xi_{j}^{2}+d^{2}\left(X_{j-1}\right)\right) \mathbb{E}\left(\mathbb{I}_{\left[b_{j},+\infty[\right.}\left(\left|Z_{j}\right|\right) \mid \mathcal{F}_{j-1}\right)
$$


and observe that

$$
\begin{aligned}
\mathbb{E}\left(\mathbb{I}_{\left[b_{j},+\infty[\right.}\left(\left|Z_{j}\right|\right) \mid \mathcal{F}_{j-1}\right) & =\mathbb{E}\left(\mathbb{I}_{\left[b_{j}^{2},+\infty[\right.}\left(Z_{j}^{2}\right) \mid \mathcal{F}_{j-1}\right) \\
& \leq \mathbb{E}\left(h_{j}\left(Z_{j}^{2}\right) \mid \mathcal{F}_{j-1}\right) \\
& \leq h_{j}\left(\mathbb{E}\left(Z_{j}^{2} \mid \mathcal{F}_{j-1}\right)\right) \leq h_{j}\left(\|g\|_{\infty}\right),
\end{aligned}
$$

where $h_{j}$ is the continuous concave function on $\mathbb{R}_{+}$such that $h_{j}(0)=0, h_{j}(x)=1$ if $x \geq b_{j}^{2}$ and $h_{j}$ linear on $\left[0, b_{j}^{2}\right]$.

Now (3.11) follows easily from (3.16), (3.17) and $\lim _{j \rightarrow \infty} h_{j}\left(\|g\|_{\infty}\right)=0$.

ii) Suppose $H_{0}(2+\epsilon)$ is satisfied for some $\epsilon>0$. Replacing $\epsilon$ by $\frac{\epsilon}{2}$, we can suppose that $H_{0}(2(1+\epsilon))$ is satisfied. Then let $p=1+\epsilon$ and let $q$ be such that $1 / p+1 / q=1$. By Hölder's inequality for conditional expectations, we get

$$
W_{j}\left(b_{j}\right) \leq \mathbb{E}\left(Z_{j}^{2(1+\epsilon)} \mid \mathcal{F}_{j-1}\right)^{1 / p} \mathbb{E}\left(\mathbb{I}_{\left[b_{j},+\epsilon[\right.}\left(\left|Z_{j}\right|\right) \mid \mathcal{F}_{j-1}\right)^{1 / q} .
$$

The second factor in the right-hand side of (3.18) has already been considered in (3.17); it is dominated by $\left(h_{j}\left(\|g\|_{\infty}\right)\right)^{1 / q}$. The first factor is bounded (by a constant). Indeed

$$
\begin{aligned}
\mathbb{E}\left(Z_{j}^{2(1+\epsilon)} \mid \mathcal{F}_{j-1}\right)^{1 / p} & \leq \mathbb{E}\left(\left(\left|X_{j}-X_{j-1}\right|+\left|d\left(X_{j-1}\right)\right|\right)^{2(1+\epsilon)} \mid \mathcal{F}_{j-1}\right)^{1 / p} \\
& \leq\left(2^{1+2 \epsilon}\left(\left\|c_{2(1+\epsilon)}\right\|_{\infty}+\|d\|_{\infty}^{2(1+\epsilon)}\right)\right)^{1 / p}
\end{aligned}
$$

where we have used the trivial inequality $(a+b)^{2(1+\epsilon)} \leq 2^{1+2 \epsilon}\left(a^{2(1+\epsilon)}+b^{2(1+\epsilon)}\right)$ for $a, b>0$. Then we obtain (3.11) in the same way as in i).

Now if $m_{1} \neq 0$ and if $d(t)$ converges to $m_{1}$ quickly enough, we can restate the CLT in a more usual form.

3.19. Corollary. Under the hypothesis of Theorem $B$, if $K=\mathbb{R}_{+}$or $\mathbb{N}$, if $m_{1} \neq 0$ and if $d(t)-m_{1}=0\left(|t|^{-\alpha}\right)$ with $\alpha>1 / 2$, we have

$$
n^{-1 / 2}\left(X_{n}-n m_{1}\right) \stackrel{\mathcal{L}}{\longrightarrow} N\left(0, \sigma^{2}\right) \quad(n \rightarrow+\infty) .
$$

Proof. We can clearly suppose $\alpha \leq 1$. Let $\epsilon(t)=d(t)-m_{1}$. By [G1] we have $X_{k} \sim k m_{1}$ a.s. But $\lim _{n \rightarrow \infty} n^{-1 / 2} \sum_{k=a}^{n} k^{-\alpha}=0$ for all fixed $a \geq 1$. This implies

$$
\lim _{n \rightarrow \infty} n^{-1 / 2} \sum_{k=1}^{n} \epsilon\left(X_{k-1}\right)=0 \quad \text { a.s. }
$$

and the result follows immediately from Theorem B.

3.20. Remarks. a) The result of the corollary is true for a more general state space $K$ if we can prove a law of large numbers in the form $\lim _{n \rightarrow \infty} n^{-1} X_{n}=m_{1} \neq 0$ a.s.

b) If $m_{1}=0$, the result of the corollary is clearly false (consider for example the simple random walk on $\mathbb{N}$ with reflecting barrier at 0 ).

c) If $X_{n}$ is positive recurrent in $\mathbb{R}$, then $X_{n} / \sqrt{n} \rightarrow 0$ in law and Theorem $\mathrm{A}$ is a CLT for the (correlated) random variables $d\left(X_{k}\right)$. 


\section{APplication TO RANDOM WALKS}

Let $(K, *)$ be a commutative hypergroup in the sense of Jewett $([\mathrm{B} . \mathrm{H}])$ such that $K \subset \mathbb{R}$. If $K=\mathbb{R}$ with the usual topology, there is only one hypergroup structure: it is the usual additive group structure $(\mathbb{R},+)([\mathrm{Z} 2])$. On the other hand, on $\mathbb{R}_{+}$ there are many hypergroup structures and (modulo isomorphism) we know that the convolution always satisfies the following property $([\mathrm{Z} 2])$ :

$$
\operatorname{Supp}\left(\delta_{x} * \delta_{y}\right) \subset[|x-y|, x+y] \quad(\forall x, y \in K) .
$$

( $\delta_{x}$ is the Dirac measure at point $x$ and Supp denotes support.) In most known examples of the discrete case (i.e. $K=\mathbb{N}$ ) the convolution also satisfies condition (4.1). Chébli-Trimèche (C-T) hypergroups (resp. polynomial hypergroups) are typical examples of the continuous case (resp. the discrete case). Let us recall briefly how they are defined.

4.2. C-T hypergroups. Let $A$ be an increasing unbounded differentiable function on $\mathbb{R}_{+}$such that $A(0)=0$. We suppose $A^{\prime} / A$ decreasing on $\mathbb{R}_{+}^{*}, \lim _{x \rightarrow+\infty}\left(A^{\prime} / A\right)(x)=$ $2 \rho \geq 0$ and $\left(A^{\prime} / A\right)(x)=\alpha x^{-1}+B(x)$ in a neighbourhood of $x=0$, where $\alpha>0$ and $B$ is a odd $C^{\infty}$ function on $\mathbb{R}$. Consider the differential operator $L=\partial_{x}^{2}+$ $\left(A^{\prime} / A\right)(x) \partial_{x}$. We know $([\mathrm{C}])$ that there is a unique hypergroup structure $\left(\mathbb{R}_{+}, *\right)$ on $\mathbb{R}_{+}$with unit $\delta_{0}$, involution the identity function and such that for every even $C^{\infty}$ function $f$ on $\mathbb{R}$, the function $u(x, y)=\left\langle\delta_{x} * \delta_{y}, f\right\rangle$ is the solution of the hyperbolic Cauchy problem $L_{x} u=L_{y} u$ with initial conditions $u(x, 0)=f(x)$ and $\partial_{y} u(x, 0)=$ 0 . Moreover the convolution satisfies property (4.1). This hypergroup is called the C-T hypergroup associated to the function $A$. Its Haar measure is $\lambda(d x)=A(x) d x$. Particular cases are the Bessel-Kingman hypergroup for which $A(x)=x^{2 \alpha+1}(\alpha>$ $-1 / 2)$ and the Jacobi hypergroup for $A(x)=(\operatorname{sh} x)^{2 \alpha+1}(\operatorname{ch} x)^{2 \beta+1}(\alpha \geq \beta>-1 / 2)$ (see [B.H] for further details).

4.3. Polynomial hypergroups. Let $p_{n}, q_{n}$ and $r_{n}$ be three sequences of real numbers such that $p_{n}>0, r_{n} \geq 0, q_{n+1}>0, q_{0}=0$ and $p_{n}+r_{n}+q_{n}=1$ for all $n \in \mathbb{N}$. The polynomials $\left(P_{n}\right)$ defined by $P_{0} \equiv 1, P_{n}(x)=x$ and

$$
x P_{n}(x)=q_{n} P_{n-1}(x)+r_{n} P_{n}(x)+p_{n} P_{n+1}(x) \quad(n \geq 1),
$$

are orthogonal polynomials with respect to some positive Radon measure $d \Pi$ on $[-1,1]$. If they have non negative linearization coefficients (i.e., for all $m, n \in$ $\mathbb{N}, P_{m} P_{n}=\sum_{r=|m-n|}^{m+n} c(m, n, r) P_{r}$ and $c(m, n, r) \geq 0$ for all $\left.r\right)$, we can define a hypergroup structure $(\mathbb{N}, *)$ on $\mathbb{N}$ by

$$
\delta_{m} * \delta_{n}=\sum_{r=|m-n|}^{m+n} c(m, n, r) \delta_{r}
$$

with unit $\delta_{0}$, and involution the identity function. It is the polynomial hypergroup with parameters $\left(p_{n}, q_{n}, r_{n}\right)$. It has convergent parameters if $\left.\lim _{n \rightarrow \infty} p_{n}=p \in\right] 0,1[$ and $\left.\lim _{n \rightarrow \infty} q_{n}=q \in\right] 0,1[$ exist. Most classical families of orthogonal polynomials on $[-1,1]$ like Jacobi polynomials give rise to a polynomial hypergroup (see $[$ B.H] for further details). 
4.4. ADC hypergroups. The two previous examples belong to a class of hypergroups we have introduced in [G4]. Let $(K, *)$ satisfy $(4.1)$ and consider the following functions on $K \times K$ :

$$
\begin{gathered}
d(t ; x)=\int_{E}(u-t) \delta_{t} * \delta_{x}(d u), \\
c_{2}(t ; x)=\int_{E}(u-t)^{2} \delta_{t} * \delta_{x}(d u) .
\end{gathered}
$$

If for all $x \in K$, the limits $\lim _{|t| \rightarrow \infty} d(t ; x)=m_{1}(x)$ and $\lim _{|t| \rightarrow \infty} c_{2}(t ; x)=m_{2}(x)$ exist (in $\mathbb{R}$ ), we say that $(K, *)$ has the property of Asymptotic Drift for the Convolution (ADC) at infinity. More briefly, we say that $(K, *)$ is a ADC hypergroup. The functions $m_{i}(i=1,2)$ are the moment functions of the hypergroup. C-T hypergroups (resp. polynomial hypergroups with converging parameters) are ADC hypergroups with moment functions given by ([G4]):

$$
\begin{gathered}
m_{1}(x)=2 \rho \int_{0}^{x}(A(t))^{-1} \int_{0}^{t} A(\xi) d \xi d t \\
m_{2}(x)=\int_{0}^{x}\left(\int_{u}^{x}(A(z))^{-1} d z\right)\left(2+4 \rho m_{1}(u)\right) A(u) d u
\end{gathered}
$$

$$
\begin{aligned}
& m_{1}(n)=(p-q) P_{n}^{\prime}(1), \\
& \left.m_{2}(n)=(p+q) P_{n}^{\prime}(1)+(p-q) P_{n}^{\prime \prime}(1)\right) .
\end{aligned}
$$

4.5. Random walks. A random walk with law $\mu \in M_{1}(K)$ on the hypergroup $(K, *)$ is a homogeneous Markov chain on $K$ with Markovian kernel given by

$$
P(x, d y)=\left(\delta_{x} * \mu\right)(d y)
$$

(see [B.H] or [G2]). For example random walks on the Bessel-Kingman hypergroup have been studied in $[\mathrm{K}]$.

If $\mu$ has finite second moment (i.e., $\int_{K} x^{2} \mu(d x)<+\infty$ ) we will say more briefly that the random walk $\left(X_{n}\right)$ has a finite second moment.

4.5.2. Proposition. Every random walk with finite second moment on a ADC hypergroup $(K, *)$ satisfies the hypothesis $H_{1}$ and $H_{2}$ of section 1.

Proof. With the notations of section 1, the functions $d$ and $c_{2}$ are given by

$$
d(t)=\int_{K}(u-t) \delta_{t} * \mu(d u)
$$

and

$$
c_{2}(t)=\int_{K}(u-t)^{2} \delta_{t} * \mu(d u) .
$$

Using the bilinearity of the convolution and notations (4.4.1) and (4.4.2), we get

$$
d(t)=\int_{K} d(t ; x) \mu(d x) \quad \text { and } \quad c_{2}(t)=\int_{K} c_{2}(t ; x) \mu(d x) .
$$

Note that these functions are well-defined because by condition (4.1) we have $|d(t ; x)| \leq|x|$ and $c_{2}(t ; x) \leq x^{2}$ for all $t \in K$. These facts allow us to use the 
dominated convergence theorem to obtain immediately $\lim _{t \rightarrow \infty} d(t)=\int_{K} m_{1}(x) \mu(d x)$ and $\lim _{t \rightarrow \infty} c_{2}(t)=\int_{K} m_{2}(x) \mu(d x)$, and the proof follows.

4.5.3. Proposition. Every random walk $\left(X_{n}\right)$ with finite second moment on a $A D C$ hypergroup $(K, *)$ satisfies condition $H_{3}$ of section 1 . More precisely we can construct $\left(X_{n}\right)$ on an adequate probability space in order that $H_{3}$ be satisfied.

Proof. We will use the tool of randomized sums introduced in $[\mathrm{K}]$ and $[\mathrm{B}]$ and formalized in [Z2] for the case of random walks on hypergroups. For simplicity we use the approach of [G2] (p. 142). Let $\left(\xi_{i}\right)$ be a sequence of i.i.d $K$ valued random variables with common law $\mu$ (= the law of the random walk) and let $\left(Z_{i}\right)$ be an independent sequence of i.i.d uniformly distributed random variables on $[0,1]$. Fix $x \in K$ and define inductively $\left(X_{n}\right)$ by $X_{0}=x$ and for every $n \geq 1$ :

$$
\left.\left.X_{n}(\omega)=\inf \left\{t ; \delta_{X_{n-1}(\omega)} * \delta_{\xi_{n}(\omega)}(]-\infty, t\right]\right) \geq Z_{n}(\omega)\right\} .
$$

By construction and according to property (4.1) of the convolution, we have that $\left|X_{n}-X_{n-1}\right| \leq \xi_{n}$ a.s. Moreover it follows from [G2] (section 2.2) that the process $\left(X_{n}\right)$ defined by (4.5.4) is a random walk of law $\mu$ on $(K, *)$ starting from $x$.

In order to apply the CLT to random walks we need some conditions on $\mu$.

4.5.5. Definitions. a) The probability measure $\mu$ on $(K, *)$ is adapted if the smallest closed subhypergroup generated by supp $\mu$ is equal to $K$.

b) The probability measure $\mu$ is spread out with respect to the Haar measure $\lambda$ of $(K, *)$ if there exists a $p$ convolution power of $\mu$ non singular to $\lambda$ (i.e., there exists $p \in \mathbb{N}^{*}$, a constant $c>0$ and an open set $V \subset K$ such that $\mu^{* p} \geq c \mathbb{I}_{V} \lambda$ ).

4.5.6. Proposition (CLT for random walks). Let $\left(X_{n}\right)$ be a random walk with law $\mu$ on a $A D C$ hypergroup $(K, *)$. Suppose $\mu$ has a finite second moment and is adapted and spread out on $K$. Then $\left(X_{n}\right)$ satisfies Theorem $B$.

Proof. By [G.G] (Theorem 3.6) we know that $\left(X_{n}\right)$ is either transient or Harris recurrent with respect to Haar measure $\lambda$. So by Propositions 4.5.2 and 4.5.3 the hypothesis of the CLT of section 2 are satisfied. To finish the proof we have to verify that $\left(X_{n}\right)$ is never positive recurrent on ADC hypergroups, i.e., Haar measure has always infinite mass. But this is an easy consequence of the structural hypothesis 4.1 .

4.5.7. Remarks. a) The adaptation hypothesis on $\mu$ is a sort of irreducibility condition for the Markov chain $\left(X_{n}\right)$; the spread out hypothesis ensures good ergodic properties for $\left(X_{n}\right)$ (see [G.G] for further details).

b) The probability measure $\mu$ is spread out in particular when some convolution power of $\mu$ has a density with respect to Haar measure. For example, in a C-T hypergroup, if for all $x$ and $y \in \mathbb{R}_{+}$the measure $\delta_{x} * \delta_{y}$ is absolutely continuous with respect to Lebesgue measure on $\mathbb{R}_{+}$, i.e., if there exists a positive measurable function $W(x, y, t)$ such that

$$
\delta_{x} * \delta_{y}(d t)=W(x, y, t) d t
$$

then $\delta_{x} * \delta_{y}$ is also absolutely continuous with respect to Haar measure $\lambda(d x)=$ $A(x) d x$ (indeed $A(x)>0$ ). It is then easily seen that for every probability measure $\mu \in M_{1}\left(\mathbb{R}_{+}\right), \mu^{* 2}$ is absolutely continuous with respect to $\lambda$ and therefore $\mu$ is spread 
out. Property (4.5.8) is satisfied for Bessel-Kingman hypergroups $[\mathrm{K}]$ and Jacobi hypergroups [F.K], and more generally if $A$ satisfies some regularity conditions in a neighbourhood of zero (see [B.S] for details).

c) In the discrete case (i.e., $K=\mathbb{N}$ ) the spread out hypothesis is automatically satisfied for every probability measure ([G.G]).

\section{REFERENCES}

[B] N.H. Bingham Random walk on spheres. Z. Wahrscheinlichkeitstheorie und verw. Gebiete 22 (1972), 169-192. MR 46:4615

[Br] B.M. Brown Martingale Central Limit Theorems Annals of Math. Stat. V 42 (1971), p. 59-66. MR 44:7609

[B.H] W.R. Bloom, H. Heyer Harmonic Analysis of Probability Measures on Hypergroups. Walter de Gruyter. Berlin-New York (1995). MR 96a:43001

[B.S] B.L.J Braaksma, H.S.V. de Snoo Generalized translation operators associated with a singular differential operator. Lecture notes in Math 415 (1974) Springer Verlag, p. 62-77. MR 54:10898

[C] H. Chébli Opérateurs de translation généralisée et semi groupes de convolution. Lecture Notes in Math 404 (1974), p. 35-59. MR 51:10745

[F.K] M. Flensted-Jensen, T. Koornwinder The convolution structure for Jacobi function expansions. Arkiv för Math. 11 (1973) p. 245-262. MR 49:5688

[G1] L. Gallardo Une loi des grands nombres pour certaines chaînes de Markov à dérive asymptotiquement stable et applications (with an abridged English version). C.R. Acad. Sci. Paris, t. 318, Série I, (1994) p. 567-572. MR 94m:60133

[G2] L.Gallardo Asymptotic behaviour of the paths of random walks on some commutative hypergroups. Contemporary Math. Volume 183 (1995) p. 135-169. MR 96d:60012

[G3] L. Gallardo Chaînes de Markov à dérive stable et loi des grands nombres sur les hypergroupes. Ann. Inst. Henri Poincaré Vol $32 \mathrm{n}^{\circ}$ 6, 1996, p. 701-723. CMP 97:05

[G4] L. Gallardo Asymptotic drift of the convolution and moment functions on hypergroups. Math. Zeitschrift., Vol. 224, nº 3, 1997, p. 427-444. CMP 97:09

[G.G] L. Gallardo, O. Gebuhrer Marches aléatoires et hypergroupes. Expositiones Math. 5 (1987) $n^{\circ}$ 1, p. 41-73. MR 89g:60024

[H.H] D. Hall, C. Heyde Martingale limit theory and its applications. Academic Press (1980).

[K] J.F.C. Kingman Random walks with spherical symmetry. Acta Math. 109 (1963) p. 11-53. MR 26:7052

[R] D. Revuz Markov chains. North Holland (1975) MR 54:3852

[Z1] H. Zeuner One dimensional hypergroups. Adv. in Math. 76 (1989) $n^{\circ} 1$ p. 1-18. MR 90i: 43002

[Z2] H. Zeuner Laws of large numbers for hypergroups on $\mathbb{R}_{+}$Math. Ann. 283 (1989) $n^{\circ} 4$, p. 657-678. MR 90j:60016

Departement de Mathematiques, Université de Tours, Faculté des Sciences et Techniques, Parc de Grandmont, 37200 Tours, France

E-mail address: gallardo@univ-tours.fr 